\title{
LA COOPERACIÓN MUNICIPAL AL DESARROLLO ANTE LA CRISIS ACTUAL
}

\author{
MUNICIPAL COOPERATION FOR THE DEVELOPMENT IN THE CURRENT CRISIS
}

Manuel Rodríguez Maciá

Universidad de Alicante. España/Spain manuelrodriguezmacia@gmail.com

Recibido/Received: 06/11/2012

Modificado/Modified: 20/01/2013

Aceptado/Accepted: 16/03/2013

\section{RESUMEN}

La crisis económica es responsable de recortes destinados por el Estado a políticas de cooperación internacional, en sus diversas administraciones. España ha invertido muchos recursos en cooperación, pero ha faltado un discurso de entender ésta como eje de la vida política. La cooperación municipal es una de las particularidades de la cooperación española. El municipio, como escuela de ciudadanía, debe formular la cooperación como parte esencial del discurso político, su visión de cercanía, de conocimiento de los problemas concretos, es esencial en aras de la eficacia. La cooperación como necesidad para desarrollar nuestra vocación humana; hay que destruir el cerco de la xenofobia. Optar por la cooperación, es hacerlo por la civilización ante la barbarie. La crisis económica es una oportunidad para revisar las mejoras que precisa el sistema de cooperación. Sugerencias a partir de la experiencia: asistencias técnicas, ayudas al desarrollo institucional, fortalecimiento de las Asociaciones Nacionales de Municipios, etc.

\section{PALABRAS CLAVE}

Cooperación internacional, política exterior, experiencias España-Iberoamérica, asistencias técnicas, fortalecimiento institucional municipal, identidad y universalidad.

\section{SUMARIO}

1. Introducción. 2. El municipio es cooperación. 3. El municipio, escuela de ciudadanía. 4. El municipio: identidad y universalidad. 5. La cooperación, vocación humana. 6. Algunas sugerencias a partir de la experiencia. 7. Epílogo: Aprender de la cooperación internacional. Bibliografía.

\footnotetext{
ABSTRACT

The economic crisis is responsible for the cuts destined by the State to policies of international cooperation in its various administrations. Spain has invested significant resources in cooperation but a speech to understand this cooperation as a key concept of the political life has been missing. Municipal cooperation is one of the peculiarities of Spanish cooperation. The municipality as a school of citizenship must develop cooperation as an essential part of the political discourse. Its close vision, knowledge of specific problems, is essential in the light of efficiency. Cooperation as a need to develop our human vocation, the siege of xenophobia must be destroyed. Opting for cooperation means opting for civilization and opposing barbarity. The economic crisis is an opportunity to revise the improvements required by the system of cooperation. Suggestions from experience: technical assistance, institutional development aid, strengthening of the National Associations of Municipalities, etc.
} 


\section{KEYWORDS}

International cooperation, foreign policy, Spain-Latin America experiences, technical assistance, institutional municipal strengthening, identity and universality.

\section{CONTENTS}

1. Introduction. 2. Municipality is cooperation. 3. Municipality, school of citizenship. 4. Municipality: Identity and universality. 5. Cooperation, human vocation. 6. Some suggestions from experience. 7. Epilogue: Learning from the international cooperation. References.

\section{INTRODUCCIÓN}

La posibilidad de continuar con las políticas de cooperación internacional al desarrollo es un debate de actualidad. Esta es una cuestión que afecta tanto al Estado en sus diversas administraciones: central, autonómica, como la local, diputaciones y municipios y a la sociedad en general que por medio de las diversas ONG manifiestan su compromiso con la cooperación internacional. A lo largo de los últimos meses son varios los foros que se han celebrado al respecto; los organizados por el Fons Català de Cooperació al Desenvolupament en noviembre del 2011, las realizadas en mayo de 2012 por la Fundación Carlos Amberes o las de junio del 2012 en la Universidad Pública de Navarra, son ejemplo de ello.

De las diversas entidades administrativas señaladas me voy a centrar fundamentalmente en la administración municipal, una de las peculiaridades más significativa de la cooperación internacional española, aunque será necesario aludir a los demás agentes antes citados.

Sobre la ayuda a la cooperación internacional han recaído en nuestro país las mayores disminuciones presupuestarias. Desde el inicio del reconocimiento de la crisis por parte del anterior gobierno, se llevaron a cabo importantes recortes en los presupuestos destinados a cooperación, que se han ido acentuando con las últimas medidas tomadas por el actual gobierno, sirva como ejemplo que la Agencia Española de Cooperación Internacional al Desarrollo [AECID] ha visto reducido su presupuesto en más de un setenta por ciento. Esta política de austeridad también es llevada a cabo por la mayoría de comunidades autónomas y las entidades locales. No faltan aquellos a quienes los recortes en los programas de cooperación, ni siquiera les merecen ningún tipo de comentario, inmersos como estamos en una situación tan grave, en la que muchas familias carecen de lo más elemental y en la que ya estamos percibiendo la falta de recursos en los servicios más básicos. Sin embargo, el debate es sumamente necesario. Entiendo que debe replantearse nuestra política de cooperación, pero lo que no podemos hacer es liquidarla. Nos va mucho en ello, entre otras cosas, la propia imagen de España como un país solidario, imagen ésta que se había afianzado en algunas regiones del mundo en los últimos años.

Ciertamente, la ayuda al desarrollo por parte de los países donantes, en general ha descendido, no obstante, hay ejemplos de lo contrario, como es el caso de Portugal, que incluso en la situación económica en la que se encuentra, ha decido mantener básicamente el presupuesto de la ayuda a la cooperación (Corrreia, 2011).

Al gobierno, el recorte en la ayuda al desarrollo no le es nada costoso medido en términos de votos o de popularidad, y así aunque en los últimos años la ayuda económica había aumentado considerablemente, en nuestra sociedad no se afianzó la idea de que la solidaridad debe extenderse más allá de nuestras fronteras (Torreblanca, 2012). 
Ciertamente en los últimos años se ha invertido mucho esfuerzo y dinero por parte de España en la cooperación, pero quizás ha faltado un discurso en el que ésta se haya planteado como un eje fundamental de la vida política y no como un hecho circunstancial propio de épocas de bonanza económica. Además, no se ha planteado la cooperación como una política de Estado en la cual participen los diversos partidos políticos, y que tenga su arraigo en la ciudadanía. Ello lleva consigo que en momentos de dificultad como los presentes, no se percibe la cooperación como una necesidad, como uno de los ejes de la política exterior, sino como algo prescindible. Es preciso que se revisen los objetivos de la cooperación, nuestras posibilidades reales en estos tiempos, las zonas prioritarias en las que debemos intervenir y todo ello con el consenso suficiente para sustentar esta política más allá de los cambios de gobierno.

\section{EL MUNICIPIO ES COOPERACIÓN}

Precisamente por ser el municipio la administración más cercana, por ser la vida municipal escuela de ciudadanía, es por lo que quiero tratar la cooperación desde el ámbito municipal y al hablar de la cooperación municipal, creo que hemos de partir de la experiencia y reivindicar lo mucho realizado por parte de los municipios, conviene poner en valor las actuaciones ejemplares que sin duda se han llevado a cabo. Reflexionar sobre la experiencia, recuperar las buenas actuaciones, es necesario en un mundo en el que prima la actuación del momento, la noticia instantánea, en definitiva, en una situación que nos acerca al abismo del olvido. Recuperar las buenas actuaciones es buscar puntos de referencia, si éstos no se tienen, nos encontramos en un mundo vacío, sin sentido. La actuación política debe basarse en una actuación ética y ésta requiere de la memoria. Y en este sentido quisiera poner de manifiesto, las actuaciones que algunos municipios españoles llevan a cabo en la restauración de lugares emblemáticos en algunas ciudades iberoamericanas, lo que sin duda contribuye a afianzar el sentido de identidad de estas localidades; como un ejemplo de tales actuaciones, podemos significar la participación que ha tenido el Fons Català de Cooperació al Desenvolupament, en la creación del Museo Municipal de Santa Tecla en el Salvador. Recuperar la memoria es parte de la restauración de nuestras ciudades y hoy más que nunca un acto de resistencia civil. Entre las resoluciones de la Asamblea de la Federación Española de Municipios y Provincias [FEMP], se pone de manifiesto la importancia que para la cooperación tiene el intercambio mutuo de experiencias y de hecho en las resoluciones aprobadas, se anuncia la creación de una base de datos de buenas prácticas (FEMP, 2011).

\section{EL MUNICIPIO, ESCUELA DE CIUDADANÍA}

Además de ser la cooperación municipal una de las peculiaridades más significativas de la cooperación internacional española, el municipio es escuela de ciudadanía y es desde ese ámbito municipal desde donde debe comenzar a formularse la cooperación como parte esencial del discurso político. Plantear el tema de la cooperación desde el municipio no es una moda pasajera, ya que la esencia del municipio es la cooperación con los otros. El municipio no se define precisamente por la frontera, sino justamente por ser un lugar de encuentro. La relación que se crea en el municipio es indudablemente la de vecindad, es decir, la de quien está al lado del otro. Es precisamente ese sentirse preocupado por los otros, 
por los vecinos, de donde surge la palabra política, justamente de la preocupación que se tiene de la polis; son preocupaciones concretas y no puras especulaciones. Es desde la instancia de lo cercano, de lo concreto, desde donde se puede llevar a cabo una restauración del valor de la política. No podemos olvidar que la política como tal, se encuentra sumamente desprestigiada en nuestro mundo y es cierto que en todo ello existen intereses muy concretos para desprestigiarla, pero también es verdad que el alejamiento de los representantes de la vida política de las preocupaciones concretas y diarias de la gente, ha ocasionado la visión de la política como una realidad esotérica, reservada para unos pocos y ha acentuado ese desprestigio de la vida política. La política tiene sentido en tanto en cuanto no es una realidad esotérica, sino doméstica; es la realidad del vivir de cada día y esa dimensión de la concreción política se lleva a cabo fundamentalmente en la vida municipal. La renovación política tiene que basarse en una actitud de vuelta a la ética y la ética no es una pura especulación, es la concreción de nuestras convicciones, de nuestros ideales, en las acciones posibles. "Es la distancia entre la naturaleza intrínsecamente ética de la toma de decisiones públicas y el carácter utilitario del debate político contemporáneo lo que explica la falta de confianza en los políticos y la política" (Judt, 2010). Es en su municipio donde el ciudadano puede encontrar la posibilidad de llevar a cabo la utopía, debido a la capacidad de incidencia que puede tener. Tal vez en el lenguaje político actual falte la dimensión utópica, en definitiva, el sentido de orientación, de finalidad y a la vez de concreción. En ocasiones se ha planteado si el perfil que deben tener los munícipes y fundamentalmente los alcaldes, debe ser el de gestor o el de político, pero entiendo que es un debate falso; tratar de la gestión de los asuntos de la polis, eso es lo que llamamos política. Con frecuencia hemos oído decir que en la ciudad no se debe hacer política, no me quiero imaginar, si los representantes de los ciudadanos no hacen política qué es lo que hacen. La figura de gestor del alcalde en la administración, se ha hecho patente en momentos de mayor centralismo. Un repaso a través de la historia de España y también de los pueblos de América así nos lo demuestra. Ocurre que a la larga, siempre en la medida que hay una mayor demanda de vida democrática, se necesita la figura política del alcalde; desde la dimensión política, es desde la que se puede ejercer el liderazgo necesario. Tal vez la celebración durante el presente año del Bicentenario de la Constitución de Cádiz y del comienzo de las independencias americanas, puede ser una buena ocasión para impulsar la cooperación municipal entre los pueblos de uno y otro lado del Atlántico. No olvidemos de una parte, la incidencia que dicha Constitución tuvo en América, la representatividad de la representación municipal en dicho texto y el papel que los municipios tuvieron en la constitución de las naciones americanas. En más de una ocasión he propuesto, en diversos foros, que uno de los pilares esenciales de esta conmemoración fuera el impulso de la cooperación municipal, pues además de ser de justicia por la historia, es necesario para el desarrollo de nuestros países, profundizar en el acercamiento de la administración a los ciudadanos, es conveniente políticamente, pues sin duda un acercamiento desde los municipios, además de sentirse más implicada la ciudadanía, elimina ciertas trabas que se pueden dar desde otras instancias del Estado.

\section{EL MUNICIPIO: IDENTIDAD Y UNIVERSALIDAD}

Toda política y fundamentalmente la municipal está en orden a la cooperación; la propia construcción de la ciudad desde el punto de vista urbanístico, está llamada a constituir un lugar de encuentro. Concebir el municipio como espacio de comunicación, como lugar de 
convivencia, es construir una ciudad, un pueblo habitable, y ello se hará realidad en la medida en que los ciudadanos se encuentren en ella como en su casa, cuando cada cual se pueda manifestar en su propia voz. En la cooperación, en la comunicación, nos sentimos pertenecientes a una comunidad, desarrollamos nuestro sentido de pertenencia, de identidad en el sentido de identificarnos con ella, de sentir que se vive en la propia casa. La identidad es una manifestación del ser comunitario. Sentir la localidad como nuestra casa es incompatible con pensar aquello de que mi casa es mi castillo. La identidad no significa la búsqueda de las diferencias con los otros, identidad significa semejanza. Sin duda, el sentido de pertenencia, de identidad, será decisivo para el éxito de la gestión municipal y para ello hemos de tener en cuenta que hay que partir de la realidad concreta, y ello nos llevará a utilizar un lenguaje más concreto y por tanto más inteligible; especialmente en la actuación política, lo concreto une, mientras el planteamiento abstracto separa. Para ello hemos de partir de la realidad y diversidad cultural de los municipios. Desde la dimensión cultural se puede captar además, cómo la identidad es la otra cara de la universalidad.

En el aspecto de la identidad tiene un papel importante el desarrollo de la ciudad histórica. La importancia de consolidar las ciudades con significación histórica, es de suma importancia para la referencia de identidad de la propia nación. Son varios los ejemplos de actuaciones que me parecen ejemplares de la cooperación española en este ámbito; quiero señalar el ejemplo del trabajo realizado en Suchitoto, en el Salvador. Una ciudad que situada en el epicentro de la guerra que vivió en el siglo XX este país, su reconstrucción se está convirtiendo en un ejemplo de la reconstrucción de la vida moral y material del país. Reconstrucción que supone no solo la restauración de sus calles, de sus plazas y de sus edificios, sino también de su proyección en el mundo de la literatura y del arte, de la restauración de su memoria y en este caso muy especial de la memoria de la guerra vivida, con la finalidad de que su recuerdo se convierta en un conjuro de esos males (Rodríguez, 2009).

La declaración de Patrimonio de la Humanidad, ya sea del patrimonio material como el inmaterial, es un ejemplo de esa expresión de cómo lo local es lo más universal. El reconocimiento del valor arquitectónico de un lugar determinado o bien las expresiones locales, como puedan ser en Guatemala la representación del Rabinal Achí, pervivencia de la cultura maya incrustada en la festividad del pequeño pueblo de Rabinal, o el Carnaval de Oruro en Bolivia o de las muchas fiestas de ciudades españolas que han adquirido este reconocimiento, es un buen ejemplo de la universalidad de lo local, de cómo la manifestación de la identidad en el fondo es lo más universal, en definitiva, lo más propio es aquello que compartimos con los otros.

La política municipal es una política de cooperación entre las ciudades y los pueblos vecinos; eso muy bien lo saben los alcaldes que desde siempre, tenían que buscar la relación a la hora de llevar a cabo un camino, la administración de las aguas o de los bosques, etc. Este sentido de la relación entre los pueblos vecinos en orden a la cooperación, recuerdo que era una de las facetas que destacaban de su función las autoridades indígenas en Guatemala. Hoy día la cooperación municipal se manifiesta por medio de una variedad de figuras asociativas en las que se enmarcan los municipios, el movimiento asociativo de los municipios podríamos decir que pertenece a la esencia del municipalismo. Generalmente al tratar de la mejora en la gestión de los servicios, con frecuencia se suele plantear desde la perspectiva de ahorro económico que ello lleva consigo la gestión mancomunada, y ciertamente es un tema que no debemos obviar, pues la sostenibilidad económica es esencial, pero también es verdad, que no solo debemos ver cómo podemos sostener y mejorar los 
servicios que poseemos, sino también las perspectivas nuevas que se abren cuando los temas se afrontan desde la mirada amplia de la cooperación con otros municipios y que pueden suponer las creación de una serie de servicios, que desde un punto de vista meramente localista no se podrían tener. La relación de cooperación entre los municipios es hoy necesaria para incluso la propia planificación del propio municipio.

En la medida que el municipio se fortalece lo hace también el Estado, pues la labor del municipio y la labor del Estado no difieren esencialmente en sus objetivos; la problemática es la misma, pero ocurre que desde el municipio las cosas se ven con una óptica de cercanía, de un mejor conocimiento de la realidad. En el Foro Local Iberoamericano celebrado en la Asunción, en septiembre de 2011, se dice en la declaración final: "Que la descentralización es una estrategia de reorganización estatal que tiene por objetivo acercar la toma de decisiones en los gobiernos locales, para avanzar hacia nuevas fórmulas de gobiernos democráticos caracterizados por la proximidad a la ciudadanía, que permita su participación efectiva y el avance de la equidad social y territorial, la cohesión social y el bienestar, al tiempo que fortalece la unidad del Estado en nuestros países". Ortega decía que el municipio solo empieza a vivir públicamente, cuando sale fuera de su propio término, transmigra a otros municipios, trata y contrata, y se ayunta con ellos, a fin de emprender algo (Ortega, 1943).

Los municipios que prosperaron a lo largo del tiempo fueron aquellos que estaban situados en el cruce de caminos de los pueblos. Los municipio han sido siempre lugares de intercambio, y al tiempo que se intercambiaban las mercancías, también lo hacían los sentimientos, las ideas, las creencias. De ahí la importancia de potenciar la relación entre los municipios fronterizos.

El municipio como antes he indicado no se define por la frontera sino por ser un lugar de encuentro. En muchos lugares podemos analizar la influencia que tienen los municipios transfronterizos, incluso como soluciones a los problemas que el Estado no sabe resolver. Tenemos ejemplos en España, como los tenemos en diversos lugares de América. Entre ellas podemos citar las de los municipios del norte Costa Rica con los municipios limítrofes de Nicaragua, los municipios fronterizos de Guatemala y el Salvador, etc. Ejemplos éstos en los que la Cooperación Internacional de los municipios españoles ha tenido gran incidencia.

Al municipio todavía es frecuente designarle en la lengua catalana como la "Universitat", lo universal, y es ciertamente en estos momentos de globalización, donde se manifiesta el rostro humano de esa globalización. Es en las calles de nuestros municipios, donde se resuelve con mayor o menor fortuna el problema de la integración de las personas que vinieron de otros lugares. La ciudad es ciudad en tanto sea más cosmopolita. Desde ese punto de vista cosmopolita, es necesario impulsar la cooperación internacional entre los municipios. En la pluralidad de acciones emprendidas por el Fons Català en la reconstrucción de Haití, por el Fons Valencià en el fortalecimiento institucional municipal en El Salvador, por los municipios andaluces en la zona norte de Marruecos, o las actuaciones de ayuntamientos como el de Alcalá de Henares en la formación de técnicos municipales en diferentes municipios de Centroamérica, son algunos ejemplos de esta dimensión de universalidad de los municipios.

\section{LA COOPERACIÓN, VOCACIÓN HUMANA}

No vamos a realizar aquí una historia detallada de la relación de los municipios con el exterior, pero sí quiero hacer la observación de la importancia que tiene el hecho de que la 
cooperación internacional forme parte del discurso de la vida política local, por cuanto de algún modo es expresión de que la acción exterior, claramente la política exterior, incumbe a todos los ciudadanos y no a un grupo de "selectos". En definitiva, la política exterior debe estar sometida al control de los ciudadanos. No deja de ser contradictorio que en estos momentos en que la situación de crisis que padecemos tiene unas causas y unas repercusiones más allá de nuestras fronteras, estemos tan ensimismados en la perspectiva interior. La ausencia de la política exterior en el debate político en la última campaña electoral a nivel nacional, como también lo fue en la anterior, es muy sintomática. Es curioso que en estos momentos en que tanto estamos hablando de la crisis mundial, nos repleguemos en las fronteras interiores. Es un problema que no aprendamos de lo ya ocurrido en otras épocas. El poeta Miguel Hernández, en su obra El hombre acecha, nos describe con la intensidad que lo puede hacer un poeta, las respuestas que el hombre puede dar, si ante las dificultades del presente, se refugia en la cueva impulsado por el miedo, o por el contrario, sabe compartir el pan con los otros. En el primer caso se convierte en fiera, en el segundo se proyecta su vocación humana.

"Por hambre vuelve el hombre sobre los laberintos/ donde la vida habita siniestramente sola./ Reaparece la fiera, recobra sus instintos,/ sus patas erizadas, sus rencores, su cola" (Hernández, 1976).

Para desarrollar nuestra vocación humana es necesaria la cooperación, para ser hombres, para ser personas.

Sin duda en estos momentos, lo primero es romper el cerco que nos imponemos, el miedo, la vuelta a la cueva, o las nostalgias de los paraísos perdidos. En estos momentos es necesario plantear la necesidad vital de la cooperación, incluso como una vuelta a un sano egoísmo. Es necesario destruir el cerco de la xenofobia, del racismo. En definitiva, en los momentos de crisis, optar por la cooperación, es hacerlo por civilización ante la barbarie.

Es cierto que vivimos tiempos de escasez, y no solo eso, sino que parece que se nos quiere imponer una visión totalmente insolidaria de la vida; resulta que todo se basa en cuánto tenemos que recortar; ni siquiera se plantea, cómo se puede hacer esto o aquello más rentable.

Los momentos de crisis pueden ser también de crecimiento. Hemos de revisar cuál ha sido nuestro comportamiento en tiempos recientes, en muchas cuestiones y en concreto, en éstas de la cooperación internacional. La actual crisis económica puede convertirse en una oportunidad para revisar y profundizar las mejoras que precisa el sistema de cooperación y en concreto, la cooperación municipal. La necesidad agudiza el ingenio y es necesario que cada euro invertido en la cooperación tenga el mayor impacto directo en el desarrollo de los pueblos.

Hay que resaltar la labor llevada a cabo por los ayuntamientos. Es necesario señalar lo mucho y bueno realizado, sobre todo en unos momentos en que parece que nos invade el más negro pesimismo. Hay que poner de manifiesto cómo la cooperación internacional ha ayudado a formar ciudadanos más conscientes y más comprometidos por un mundo más solidario, lo que es una magnifica inversión. No es un tema cuantificable, pero sin duda el despertar a la solidaridad con los países más pobres, ha llevado a que seamos más solidarios entre nosotros y esto tiene especial significación, ahora que en el interior de nuestros pueblos y ciudades se ha agrandado la división entre los que poseen y los que carecen de lo más elemental. En el haber de la cooperación municipal hemos de destacar esa concreción, esa visión de cercanía que está presente desde la perspectiva del municipio y ello se traslada al mundo de la cooperación internacional, haciéndola más eficaz por ese sentido de cercanía y 
de un conocimiento más concreto de las necesidades y de la realidad de la gente. Es fundamental que la cooperación forme parte de la dimensión ciudadana y no se convierta solamente en una dimensión "altamente profesionalizada", fuera de la realidad política, pues de hecho si la desarraigamos de la vida política, le hurtamos a la larga la sostenibilidad.

En su inicio, la ayuda al desarrollo estaba muy enfocada en su dimensión económica, con el objetivo del crecimiento económico, sin embargo, a partir de los años 90 surgió a instancias del Programa de las Naciones Unidas para el Desarrollo [PNUD] el concepto de desarrollo humano, y a la cooperación se le añadieron nuevos objetivos como la equidad de género, la sostenibilidad ambiental, la lucha contra la corrupción, la defensa de la democracia, el fortalecimiento de la paz, etc., y en ese marco, el municipio tiene un papel fundamental, pues siempre el municipio se ha caracterizado, tal y como afirmaba Adolfo Posada, por buscar el bienestar, la libertad de las personas y la paz (Posada, 2007). A partir de la mitad de los años 90, ante el debate originado respecto a la eficacia de la ayuda, se puso el acento en la importancia del marco institucional y en ello tiene un papel primordial el fortalecimiento de la institución municipal.

Tenemos que ser también autocríticos. A lo largo de estos años hemos de señalar los aciertos y reconocer los errores cometidos, entre éstos creo que nos ha faltado una mayor coordinación entre las entidades cooperante, me refiero tanto a la cooperación municipal, como también a la coordinación entre ésta y las diversas instancias del Estado y con otros organismos internacionales. En algunas zonas es excesivo el número de actores de la cooperación que están realizando las mismas actuaciones; es esencial la coordinación entre todas ellas, pues aparte del dispendio económico que todo ello conlleva, se convierte en una mala práctica, la inflación por ejemplo de cursos que se ofrecen y en muchas ocasiones da lugar a la creación de la figura de funcionarios, sean técnicos o cargos electos, que practican el nomadismo a través de las múltiples actividades de formación que se imparten.

Asimismo creo que sin proponérnoslo, hemos creado una serie de disfunciones en aquellos países con los que cooperamos, pues hemos creado una nueva clase privilegiada, la de ciertos cooperantes, de acá y de allá. Quizás nos hemos conformado evaluando lo que dábamos y no el resultado de nuestras acciones, nuestras alianzas con nuestras contrapartes, el que ellos se apropiaran del trabajo, única manera de que pueda arraigar y permanecer.

Es necesario plantear la política de cooperación internacional, como una política de Estado, fruto de un pacto entre los diversos partidos del arco parlamentario y en coordinación con todas las administraciones del Estado y con los agentes no gubernamentales de la cooperación.

\section{ALGUNAS SUGERENCIAS A PARTIR DE LA EXPERIENCIA}

A partir del trabajo en cooperación que he llevado a cabo en diversos países y especialmente en los de Centroamérica y el Caribe, quiero destacar la importancia que ha tenido la transferencia de saberes, la puesta en común de experiencias entre los municipios de los diversos países; este ha sido uno de los ejes fundamentales en el trabajo de la cooperación, y que desde mi punto de vista ha tenido mejores resultados, además de constituir una posibilidad muy factible desde el punto de vista de la viabilidad económica. A título de ejemplo indico los siguientes:

a) Los planes urbanísticos y de delimitación de la zona marítimo-terrestre en Guanacaste en Costa Rica. 
b) La creación de la unidad tributaria de la zona de los Santos de Costa Rica.

c) La unidad técnica vial de la zona del Pacífico en el mismo país.

d) La creación de empresas municipales de aguas en diversas mancomunidades de Guatemala.

e) Las asistencias técnicas para la municipalización del servicio de aguas en Honduras, en los municipios de La Paz, Villanueva etc.

f) La asistencia técnica para la puesta en práctica de una biblioteca de estudios municipales en Costa Rica.

Me parece también de especial importancia destacar en el aspecto del fortalecimiento institucional municipal:

a) El trabajo realizado respecto a la creación o en su caso, el fortalecimiento de las asociaciones nacionales de municipios.

b) Dar a conocer las experiencias en la creación, el desarrollo y el funcionamiento de las diversas figuras asociativas en los municipios de los diversos países claro está, en ello como en todo, sin mimetismos.

c) Fomentar la pluralidad partidaria en el interior de las asociaciones de municipios.

d) El trabajo realizado para la consecución de la elección de los alcaldes en Costa Rica y Panamá.

e) El apoyo para lograr una mayor participación de la mujer en el gobierno local.

f) El trabajo relativo a la creación de la carrera administrativa municipal.

g) Cooperación en la formación en ciclos cortos, para los cuales se pueden aprovechar las asistencias técnicas.

h) Apoyo a cursos de formación de ciclos más largos como puedan ser los diplomados, los másteres, etc. Para realizar este tipo de actuaciones, es necesaria la alianza con universidades, institutos de investigación etc. Y será muy conveniente que dichas alianzas se establezcan en el mayor grado posible, con las instituciones de los países en los que se vaya a realizar esta actuación. Naturalmente los socios fundamentales deben ser los propios municipios, que actuarán por medio de las asociaciones de municipios. Es básico para el aprovechamiento de los mismos cursos, para la sostenibilidad de los mismos, que los municipios se apropien de ello.

Quiero también resaltar el trabajo realizado con los diversos partidos políticos, el asesoramiento a los propios partidos políticos, el asesoramiento antes las nuevas leyes, etc., y en especial considero que se debe incidir en introducir en la filosofía de los partidos políticos la dimensión municipal; ello hará que los mismos tengan un mayor arraigo, por cuanto plantearse los temas del municipio les hará estar más próximos a la ciudadanía. Entre los resultados que de este trabajo se pudo obtener, quiero señalar la creación en todos los países de Centroamérica de las comisiones parlamentarias de asuntos municipales. Volvemos pues al principio, a través del municipalismo se redescubre la dimensión política de la cooperación. El Municipio, decía Joan Rigol, es un modo de reivindicar la primacía de la política (Rigol, 2001).

Al principio de este artículo, hacía referencia a cómo el municipio fortalece la identidad, entendida ésta, no como a veces lo planteamos, como la búsqueda de las diferencias, sino desde el punto de vista de apropiarse de la propia ciudad, de sentirse partícipe de la misma y ello comporta un modo propio de gestionar la ciudad, de creación de un tipo determinado de ciudad, en definitiva, en la creación de ciudades habitables, de entender la ciudad como lugar de encuentro y cómo ese modelo, en la medida que el ciudadano se siente identificado con él, es un elemento fundamental para el éxito de la gestión. La ciudad habitable es una ciudad 
segura. En gran parte el problema de la inseguridad en muchos países, es un lastre para su desarrollo en todas las facetas y conviene que tenga un tratamiento desde el propio municipio; la cooperación tiene un papel que jugar y creo que algunas de las experiencias que se han llevado a cabo en Centroamérica son sumamente alentadoras. Me encuentro totalmente identificado con las palabras de José Ángel Sotillo cuando indica la importancia de las buenas prácticas de los proyectos que fortalecen la autoestima, lo cual es de gran importancia en contextos donde hay mucha violencia (Sotillo, 2011). Ejemplos como los llevados a cabo en la ciudad de Santa Tecla en El Salvador, o en la Ceiba en Honduras, en los que se ha realizado una política de seguridad desde los municipios, desde la base de crear ciudades habitables, son experiencias sumamente interesantes y a tomar en consideración.

\section{EPÍLOGO: APRENDER DE LA COOPERACIÓN INTERNACIONAL}

A lo largo de esta exposición en la que he intentado plantear la cooperación en estos tiempos de crisis, he procurado hacer algunas reflexiones sobre la necesidad de continuar y profundizar en la cooperación internacional y a la vez, convertir la situación de crisis en una oportunidad para revisar y mejorar la cooperación sobre todo desde el ámbito municipal y a la vez reflexionar sobre la necesidad de enraizar dichas políticas de cooperación en el discurso municipal. Tener una visión estrictamente economicista de la cooperación parece que no tenga mucho sentido, pero también es verdad que la cooperación es una inversión que nosotros hacemos en beneficio de nuestras propias comunidades. Antes hice referencia al valor de la solidaridad alentada en la cooperación internacional. Un activo tan importante en estos tiempos, también para resolver o al menos paliar la situación en el interior. También hemos de considerar como una ganancia el hecho de que en la medida en que nos abrimos hacia el exterior, encontramos los senderos para fomentar la unión en el interior. Las visiones localistas devienen en enfrentamiento; la perspectiva amplia conduce a la concordancia. Quizás para encontrar los puntos de concordancia interior necesitamos ver el paisaje, y éste requiere siempre de una mirada externa.

Considero también la necesidad de que la cooperación con otros países debe de tener viaje de ida y vuelta. Es mucho lo que los países con los que cooperamos nos pueden aportar, y por ello creo que hemos de tener la apertura necesaria para aprender de ellos. Siempre me impresionó el respeto al medio ambiente natural que encontré en Costa Rica -lo cual no quiere decir que no existan actuaciones en nada encomiables- lo que contrasta con la devastación a la que hemos sometido nuestra costa en muchos lugares de España.

También la actuación en la gestión de muchos municipios, en los que se buscan soluciones en situaciones sumamente adversas y con una enorme escasez económica y a la vez, ejercen la representación política en muchos casos con toda dignidad.

Pienso que muchas de las buenas prácticas que desde la cooperación española se han llevado a cabo en diversos países, debiéramos potenciarlas en el nuestro; pienso entre otros ejemplos, en la potenciación de las mancomunidades y las federaciones de municipios o en los planes de desarrollo económico local. El último de los foros locales iberoamericanos celebrado del 17 al 19 de octubre en Madrid pone de manifiesto la necesidad de que los municipios, ante la situación económica, tengan un papel protagonista en el desarrollo económico, pues es en el entorno local donde se hace presente, la situación dramática del desempleo, la marginación, el deterioro de la convivencia. 
El hecho de impulsar el voluntariado entre los funcionarios municipales trasladando sus experiencias a otros países, es un estímulo para estos funcionarios que pueden desarrollar sus habilidades y aumentar por medio de los programas de cooperación su propia formación.

Y volviendo a la situación de crisis que hoy vivimos, no podemos dejar de preguntarnos, cuánto podríamos habernos evitado si hubiésemos tenido una mente abierta a las realidades de Iberoamérica. No podemos despreciar la rica experiencia del otro. Podemos aprender mucho de la trayectoria económica reciente de estos países: "El gobierno ha emprendido una política de rescate bancario, protegiendo a los bancos privados y a los banqueros que se han enriquecido con el mismo rescate. El Estado ha asumido la deuda privada y ha endeudado aún más al país" (Lombera 2001). Estas palabras no son de hoy, fueron dichas por la Coordinadora del área de desarrollo y gestión municipal de COPEVI el año 2000, en el foro celebrado en Mataró. ¿Encontramos algún parecido con la realidad de nuestros días?

Por último, no quiero al menos dejar de sugerir la perspectiva amplia que podemos captar de nuestra propia realidad mediterránea, en la apertura con los pueblos de la otra orilla y en aquel Mediterráneo más amplio, como es aquel de América. Al hablar de Iberoamérica y el Mediterráneo lo estamos haciendo de un lugar común; el mar de la latinidad es el que da nombre al espacio que modernamente llamamos Latinoamérica. Un lugar de encuentro de nuestras propias raíces culturales, con las de tantos pueblos de Europa, con el mundo indígena, de encuentro con el mundo africano; hoy día para conocer muchas de las antiguas tradiciones del África negra hemos de recurrir al mundo africano recreado en Latinoamérica; también con las emigraciones ya antiguas del Oriente Medio compuestas por las comunidades provenientes del Líbano, Siria, Palestina, las comunidades judías y las del Extremo Oriente. No sé si sabemos apreciar el valor cultural y político que supone ese amplio espacio de encuentro ¿Cuánto vale tener una visión universal? El poeta Rubén Darío habitante de varias patrias, ciudadano de la lengua, descubrió la mediterraneidad del Caribe desde la atalaya de Mallorca o de Málaga, su poesía está llena de referencias al mundo clásico de Grecia y de Roma. Unus -versus- alios. La universalidad, una respuesta a eso que llamamos la globalización. Una mirada desde el Mediterráneo nos lleva a poner la mirada en el mundo de la ciudad, desde la óptica de la ciudad, se puede vislumbrar con mayor exactitud los problemas de los hombres, desde la óptica de la ciudad vislumbramos la diversidad y a la vez la universalidad, la preocupación por la misma es lo que da nacimiento a la política. El Dr. Posada antes citado, definía la ciudad como "la síntesis sociológica más representativa de los beneficios de la paz" (Posada 2007). Cuando se firmaron los Acuerdos de Paz de Esquipulas, se establecieron como premisa para asentar la misma, la democratización y la consecución de una sociedad más equitativa, la descentralización y sobre todo, la potenciación del municipio. La cooperación española optó por esta apuesta por la paz. ¿No valdría la pena plantearnos también la cooperación municipal con los vecinos de nuestro Mediterráneo más próximo, aprovechar sin mimetismo nuestra labor de cooperación que ya es larga con Iberoamérica, en un momento en que desde las ciudades se quiere respirar el aire de la libertad? Y llevar a cabo esta acción también en compañía de aquellos municipios del otro Mediterráneo, es mucho lo que de esa larga experiencia podemos aprender.

\section{BIBLIOGRAFÍA}

CORREIA, M. (2011), Cooperación externa sortea la crisis. En: http://ipsonoticias.net/nota.asp?idn ews $=97244$ 
FEMP. Federación Española de Municipios y Provincias. Asamblea General de 24 de septiembre de 2011 (pp. 9-10). En: http://asamblea.femp.es/

HERNÁNDEZ, M. (1976), Obra poética completa: El hombre acecha. Madrid, Editorial Zero.

JUDT, T. (2010), Algo va mal. Madrid, Taurus.

LOMBERA, R. (2001), "Red de Educación Popular para la Construcción del Poder Local (REPPOL)", $2^{\circ}$ Encuentro de Municipalismo en Centroamérica, México y Caribe. Mataró, 2000. Barcelona, Confederación de Fondos de Cooperación y Solidaridad, pp. 32-37.

ORTEGA y GASSET, J. (1943), Obras completas. Madrid, Espasa-Calpe.

POSADA, A. (2007), El régimen municipal de la ciudad moderna. Madrid, Federación Española de Municipios y Provincias.

RIGOL, J. (2001), "Saludo de bienvenida", $2^{\circ}$ Encuentro de Municipalismo en Centroamérica, México y Caribe". Mataró, 2000. Barcelona, Confederación de Fondos de Cooperación y Solidaridad, pp. 1518.

RODRÍGUEZ MACIÁ, M. (2009), La ciudad lugar de encuentro. San José de Costa Rica, Fundación Desarrollo Municipal Centroamericano y del Caribe-Agencia Española de Cooperación Internacional de Desarrollo [DEMUCA-AECID]

SOTILLO, J.A. (2011), El sistema de cooperación para el desarrollo. Madrid, Catarata.

TORREBLANCA, J.I. (2012), "Tiempo de recortes". El País, 6 abril, p.4.

\section{Breve currículo}

\section{Manuel Rodríguez Maciá}

Doctor en Filosofía y Letras por la Universidad Complutense de Madrid. Profesor de Enseñanzas Medias. Profesor invitado en varias universidades. Dirigió el Centro Regional de la UNED (Universidad a Distancia) en Elche. Autor de diversos libros y numerosos artículos sobre municipalismo. Gran experiencia en la actividad política: diversos cargos electos de representación nacional, regional, provincial y local. Actividades en la Cooperación Internacional, especialmente desde el mundo local y en numerosas conferencias internacionales. Ha dirigido durante más de cuatro años la Fundación DEMUCA (Desarrollo Municipal de Centroamérica y el Caribe) a propuesta de la AECID (Agencia Española de Cooperación Internacional del Desarrollo). Miembro del Departamento de Políticas Públicas del Estado de la Universidad de Alicante. 